\title{
Design of Linear Phase Low Pass FIR Filter using Particle Swarm Optimization Algorithm
}

\author{
Neha \\ M.Tech. Research Scholar \\ ECE Department \\ SLIET, Longowal
}

\author{
Ajay Pal Singh \\ Associate Professor \\ ECE Department \\ SLIET, Longowal
}

\begin{abstract}
The everyday broadening field of signal processing has digital filters to play a major role. Linear phase FIR filters are used in vast number of applications due to their nature of phase linearity as well as frequency stability. The traditional nonoptimization methods available for filter design suffer from the problem of need for analog to digital conversion and also the inefficient control of frequency response. The conventional gradient based optimization methods are unable to solve non-differential functions and converges to local optimum solution. Thus this paper presents the evolutionary optimization technique of Particle Swarm Optimization (PSO) for the design of linear phase digital low pass (LP) FIR filter. Given the specifications of desired filter to be realized, PSO algorithm results in an optimal coefficient set for linear phase FIR filter approximating the ideal specifications. In this paper PSO algorithm is used with constriction factor approach to solve the multimodal, highly non-linear filter design problem. This method has the property of parameter independence and thus ensuring convergence while fully exploring the solution space. The velocity and position updating rules of original PSO algorithm is used for the design of low pass FIR filter of order 20. The extensive simulation results obtained from the proposed method shows superiority of the algorithm.
\end{abstract}

\section{General Terms}

Linear phase FIR, Particle Swarm Optimization technique, Non-recursive filters.

\section{Keywords}

Linear phase low pass (LP) filter, PSO algorithm, Passband, Stopband.

\section{INTRODUCTION}

Digital filters are used in wide variety of applications from signal processing, aerospace, control systems, defense equipments, telecommunications, system for audio and video processing to systems for medical applications to name just a few. Basically filter refers to a frequency selective device which extracts the useful portion of input signal lying within its operating frequency range and could be contaminated with random noise due to unavoidable circumstances. Analog filters are implemented with discrete components but the digital filters perform mathematical operations on a sampled, discrete time signal to reduce or enhance the desired features of the applied signal. Digital filers are superior to their analog counterpart due to its wide range of applications and better performance. The advantages of digital filters over analog filters are small physical size, high accuracy and reliability. Digital filtering is one of the most powerful tools of Digital Signal Processing. Digital filters are capable of performance specifications such as ability to achieve multi-rate operation and exact linear phase that would, at best, be extremely difficult, if not impossible, to achieve with an analog implementation. In addition, digital filter characteristics are easy to change under software control. Digital filters are widely used in the fields of automatic control, telecommunications, speech processing and many more. Digital filters are of two types; finite impulse response (FIR) and infinite impulse response (IIR) filter.

Traditional methods for such filter design for filter coefficient selection involve windowing functions as Butterworth, Chebyshev, Kaiser etc [1-3] for FIR filters and which can further be transformed to digital Low pass, High pass, Band pass or Band stop filter using transformation technique such as bilinear transformation for IIR filter designs. Remez exchange algorithm proposed by Parks and McClellan [4] (called PM algorithm) is better than all other traditional methods for design of FIR filters but it too has the limitation of computational complexity and high passband ripples [5]. Such filters have great errors and need optimization, i.e. minimization of mean square error between desired response and estimated filter output. Steepest-descent method of optimization [6] can approximate any kind of frequency response for linear phase FIR filter but the transition width is to be compromised which is not acceptable. The other classical gradient based optimization methods are not suitable for FIR filter optimization because of the several reasons [7]. As the filter optimization is not new and several efforts have already been made for optimum filter design. Evolutionary optimization techniques as Genetic Algorithm [8], Tabu Search [9], Artificial Bee Colony optimization [10], Differential Evolution [11] are implemented for the design of optimal digital filters. These approaches proved themselves quite efficient by providing better control of performance parameters in addition to high stopband attenuation. Heuristic optimization technique of Genetic Algorithm gives efficient results for local optimum but is not very successful in determining global optimum [12]. This paper presents the use of one of the evolutionary optimization technique called Particle Swarm Optimization (PSO) for design of digital filters with better parameter control \& to better approximate the ideal filter. The PSO technique is developed by Kennedy and Eberhart [13] simulating the social behavior of swarm of birds searching for food. This optimization method requires no gradient and achieves a global optimal solution for the given multimodal objective function minimization in digital design problems. Several modifications of the conventional PSO also exist. Luitel et al. [14] proposed the use of PSO with differential evolution for design of optimal filter. The method converges faster but with higher value of passband ripples. Quantum-behaved PSO proposed by Sun et al. [15] is another novel algorithm based on the quantum behavior of particles. 
This concept of wave nature of each particle helps in exact determination of the position and velocity vector for the particle as used in [16]. PSO using LMS and minimax strategies [17] is used for LP filter design.

But all these algorithms have results that yet need to be optimized due to their inherent problems of control parameter dependence, search stagnation or premature convergence. This paper presents an alternative to these problems by using the PSO algorithm with constriction factor approach [18]. The designed low pass FIR filter better optimizes the ideal filter characteristics while being computationally fast.

The paper outline is as follows: Section 2 defines the basic mathematics involved for the FIR low pass filter design. Section 3 presents a summary of the proposed PSO algorithm and basic steps involved. Section 4 describes the design filter specifications and the simulation results obtained. Finally section 5 briefly concludes the paper.

\section{LOW PASS (LP) FIR FILTER DESIGN PROBLEM}

The frequency response of a desired FIR filter is given as:

$H_{d}\left(e^{j \omega}\right)=\sum_{n=0}^{N} h[n] e^{-j \omega n}$

where $h[n]$ represents the impulse response of the filter. $N$ is the order of the filter with $\mathrm{N}+1$ defining the filter length. The values of $h[n]$ are determined in the design process. The approximate filter resulting from the design simulation is compared with the ideal frequency response. For a LP filter the ideal response $H_{i}\left(e^{j \omega}\right)$ is defined as:

$H_{i}\left(e^{j \omega}\right)= \begin{cases}1 & \text { for } 0 \leq \omega \leq \omega_{c} \\ 0 & \text { otherwise }\end{cases}$

where $\omega_{c}$ is the cut off frequency of the LP filter to be designed. Different types of error fitness functions have been used in different literatures over the years. A basic error function used in PM algorithm for filter design in [4] is as defined in Eq. (3) below:

$E(\omega)=G(\omega)\left[H_{i}\left(e^{j \omega}\right)-H_{d}\left(e^{j \omega}\right)\right]$

where $H_{i}\left(e^{j \omega}\right)$ is the ideal frequency response and $H_{d}\left(e^{j \omega}\right)$ is the frequency response of the designed approximate filter; $G(\omega)$ represents the weighing function used to provide independent control of the parameters $\delta_{p}$ and $\delta_{s}$ for the approximate error in the two frequency bands. For this paper $G(\omega)$ is chosen to be:

$G(\omega)= \begin{cases}\delta_{s} / \delta_{p} & \text { in the passband } \\ 1 & \text { in the stopband }\end{cases}$

The error fitness function to be minimized adopted in this paper is as given in Eq. (5). This fitness function combined with proposed filter design technique results in minimum passband and stopband ripples achieved so far by other optimization techniques.

Fitness $=\sum \operatorname{abs}(|E(\omega)|)$
The minimization of the error fitness function given in above equation is the objective of PSO algorithm used in the paper for optimization of filter performance. Unlike other fitness function that only consider the maximum value of the errors used for filter optimization, Eq. (5) involves summation of all absolute errors for both pass and stopband frequencies, thus yielding much higher stopband attenuation as well as smaller ripples in passband of the designed LP filter.

\section{PARTICLE SWARM OPTIMIZATION}

The particle swarm optimization (PSO) is a parallel evolutionary computation technique motivated by the simulation of social behavior. The PSO algorithm was first introduced by Kennedy and Eberhart [13]. The PSO algorithm is initialized with a population of random candidate solutions, conceptualized as particles. Each particle is assigned a randomized velocity and is iteratively moved through the problem space, which is, updated by applying some kinds of operators according to the fitness information obtained from given problem statement to be simulated so that the individuals of the population can be expected to reach better solution areas. It is attracted towards best fitness position obtained so far by the individual itself (cognitive part) and by the whole population (social part).

The position and velocity associated with each particle are arranged in a form of matrix. For the $i^{\text {th }}$ particle it can be given as:

$p_{i}=\left[p_{i 1}, p_{i 2}, \ldots, p_{i D}\right]$

$v_{i}=\left[v_{i 1}, v_{i 2}, \ldots, v_{i D}\right]$

The length of the vector $\mathrm{D}$ represents the dimensions of the specified problem with $(\mathrm{i}=1,2, \ldots, \mathrm{N})$ specifying the population set of the particles. A fitness function is evaluated for all swarm particles and optimization of this fitness function is the aim, like a swarm of birds flocking stops when they found the food. For the fitness function optimization the position and the velocity of the particle is updated according to the individual best and social best results (as defined above) found so far according to the equations:

$$
\begin{gathered}
v_{i d}{ }^{t+1}=\chi\left[v_{i d}{ }^{t}+c 1 * r 1() *\left(\text { pbest }_{i d}-p_{i d}\right)+\right. \\
\left.c 2 * r 2() *\left(\text { pbest }_{g d}-p_{i d}\right)\right] \\
p_{i d}{ }^{t+1}=p_{i d}^{t}+v_{i d}{ }^{t+1}
\end{gathered}
$$

The superscripts $t$ and $t+1$ denote the index of previous and next iterations, respectively. $\mathrm{c} 1$ and $\mathrm{c} 2$ are the constriction coefficients used normally to be 2.05. r1( ) and r2( ) in Eq. (8) specifies the random numbers uniformly distributed from decimal zero to one updated every time they occur. The factor $\chi$ called constriction factor is defined in [17] and is given by the formula in Eq. (10).

$\chi=\frac{2}{\left|2-\varphi-\sqrt{\varphi^{2}-4 \varphi}\right|}$, where $\varphi=c 1+c 2>4$

This velocity update equation specified in Eq. (8) uses constriction factor method and thus convergence is 
independent of the iteration cycles. For each iteration of the algorithm particles are moved according to Eq. (8) and (9) keeping within a bound of maximum velocity specified as $v_{\max }$ for the evaluation of particle fitness for this new position and velocity during the iterative process. The steps for the PSO algorithm as employed for FIR LP filter design is provided in Table 1 . The best filter coefficient value hgbest having $(\mathrm{n} / 2+1)$ elements is used for forming the complete filter response coefficients $(n+1)$ by copying them due to phase linearity of the filter. Frequency and the magnitude spectrum are generated from this complete filter coefficient vector.

\section{Table 1: Basic steps of PSO algorithm}

Step I. Specify the fixed parameters used for the design of low pass linear phase FIR filter. This includes passband edge frequency, $\boldsymbol{\omega}_{\boldsymbol{p}}=0.25$; stopband edge frequency, $\boldsymbol{\omega}_{\boldsymbol{s}}=0.3$; passband ripple, $\boldsymbol{\delta}_{\boldsymbol{p}}=0.1$; stopband ripple, $\boldsymbol{\delta}_{\boldsymbol{s}}=0.01$; order of the filter $\mathrm{n}=20$ (thus filter has a length $\mathrm{M}=21$ ).

Step II. Initialize the population of particles with, $\mathrm{N}=30$; number of dimensions (filter coefficients), $D=11 \quad(n / 2+1)$; minimum and maximum value for the population of filter coefficients, $\boldsymbol{h}_{\text {min }}=-2 ; \boldsymbol{h}_{\text {max }}=2$; velocity range, $\boldsymbol{v}_{\min }=0.01$; $\boldsymbol{v}_{\text {max }}=0.1$; number of samples taken $=128$; sampling frequency, $\boldsymbol{f}_{\boldsymbol{s}}=1 \mathrm{~Hz}$; maximum no of iterations, itmax $=250$.

Step III. Generate the initial particle vectors using above defined parameters and calculate initial error fitness value for the whole population using Eq. (3) and (5).

Step IV. Calculate the minimum fitness value from error fitness vector and compute individual best (hibest) and group best (hgbest) values from whole population .

Step V. Update the velocity and position (filter coefficient value) according to Eq. (8) and (9) while checking against the limits of filter coefficients, taking them now as the initial particle vectors; also calculate the error fitness value from these updated parameters and hibest and hgbest accordingly.

Step VI. If values of vector hibest and hgbest calculated in Step V are better than those calculated in Step IV, then replace the vectors and no change if otherwise.

Step VII. Iterate continuously from Step IV to Step VI until convergence criteria (reaching itmax or error fitness value equals minimum error fitness) is met.

\section{SIMULATION RESULTS AND DISCUSSION}

The proposed algorithm is simulated using MATLAB 7.10 for the design of LP Finite Impulse Response filter for order (n) taken to be 20. The actual filter designed used all the parameters of ideal filter defined here as:-

$$
H_{d}=\left\{\begin{array}{lc}
1 & \text { for } 0 \leq \omega \leq 0.275 \\
0 & \text { for } 0.275 \leq \omega \leq 0.5
\end{array}\right.
$$

where $\omega_{c}=0.275$ represents the cut-off frequency of the filter in hertz. From this the passband and stopband edge frequencies of the filter are calculated as:-

$$
\begin{gathered}
\omega_{p}=\omega_{c}-0.25=0.25 \\
\omega_{s}=\omega_{c}+0.25=0.3
\end{gathered}
$$

The same are used in PSO algorithm as mentioned in Table 1 with a transition width of 0.05 .The algorithm is run 100 times and the best and average results are recorded. Table 2 shows the best optimized filter coefficients obtained for designing the desired LP filter, the best coefficient value among all the 100 runs.

Table 3 represents the various performance parameters of the designed filter recorded to show its efficiency. From the table it is inferred that the designed linear phase LP FIR filter provides maximum stopband attenuation as high as $31.6 \mathrm{~dB}$ which is greater than that achieved by other results reported in Table 4 below. The maximum passband ripple is 0.0390 which is also very small. Table 3 also summarizes maximum, average, standard deviation and resulting variance for passband ripple, stopband ripple, stopband attenuation (in $\mathrm{dB}$ ) and also the execution time for the desired LP filter design using proposed method.

Table 4 shows the comparison of results of the proposed method with other reported results. The simulation results Ababneh [12] and Liu [19] are for the same filter specifications. The comparison parameters of Table 4 include maximum passband and stopband ripples, maximum stopband attenuation and the transition width.

Table 2: Optimized Low Pass FIR filter coefficients of order 20

\begin{tabular}{|c|c|}
\hline $\mathbf{h}(\mathbf{n})$ & PSO result \\
\hline $\mathrm{h}(1)=\mathrm{h}(21)$ & -0.0109749645361891 \\
\hline $\mathrm{h}(2)=\mathrm{h}(20)$ & 0.00086682618993582 \\
\hline $\mathrm{h}(3)=\mathrm{h}(19)$ & 0.023409416091128 \\
\hline $\mathrm{h}(4)=\mathrm{h}(18)$ & -0.0108519401054901 \\
\hline $\mathrm{h}(5)=\mathrm{h}(17)$ & -0.0358553459786377 \\
\hline $\mathrm{h}(6)=\mathrm{h}(16)$ & 0.0342126823588323 \\
\hline $\mathrm{h}(7)=\mathrm{h}(15)$ & 0.0471110010089426 \\
\hline $\mathrm{h}(8)=\mathrm{h}(14)$ & -0.086291975144066 \\
\hline $\mathrm{h}(9)=\mathrm{h}(13)$ & -0.0540626109210174 \\
\hline $\mathrm{h}(10)=\mathrm{h}(12)$ & 0.311320366834167 \\
\hline $\mathrm{h}(11)$ & 0.556621606901486 \\
\hline
\end{tabular}

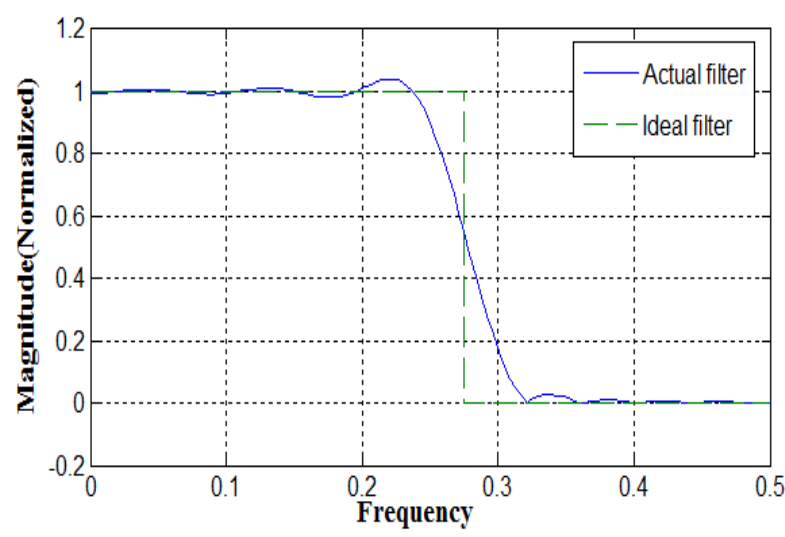

Fig. 1: Magnitude plot for FIR LP filter of order 20 
Table 3: Performance parameters of Low Pass FIR filter

\begin{tabular}{|c|c|c|c|c|}
\hline \multirow{2}{*}{ Parameters } & \multicolumn{3}{|c|}{ PSO result (for filter of order 20) } \\
\cline { 2 - 5 } & $\begin{array}{c}\text { Maximum } \\
\text { (for filter in fig. 1) }\end{array}$ & Mean & $\begin{array}{c}\text { Standard } \\
\text { Deviation }\end{array}$ & Variance \\
\hline Passband ripple & 0.0390 & 0.03799 & 0.00302488 & $9.1499 \mathrm{E}-06$ \\
\hline Stopband ripple & 0.0260 & 0.027445 & 0.003631043 & $1.31845 \mathrm{E}-05$ \\
\hline $\begin{array}{c}\text { Stopband } \\
\text { attenuation (dB) }\end{array}$ & 31.6 & 31.38586 & 1.041077 & 1.08384 \\
\hline $\begin{array}{c}\text { Execution time(250 } \\
\text { cycles) }\end{array}$ & 5.972055 & 6.048243 & - & - \\
\hline
\end{tabular}

Table 4: Comparison of various parameters of LP FIR filter from different literatures

\section{Parameters compared ( All are for LP filter)}

\begin{tabular}{l|c|c|c|c|c}
\hline \multicolumn{1}{c|}{ Reference Type } & Order & $\begin{array}{c}\text { Maximum } \\
\text { stopband } \\
\text { attenuation }(\mathbf{d B})\end{array}$ & $\begin{array}{c}\text { Maximum } \\
\text { Passband ripple }\end{array}$ & $\begin{array}{c}\text { Maximum } \\
\text { stopband ripple }\end{array}$ & Transition width \\
\hline Ababneh et al. $[12]$ & 30 & $<30$ & 0.15 & 0.031 & 0.05 \\
\hline Karaboga et al. $[11]$ & 20 & $\mathrm{NG}^{*}$ & $>0.08$ & $>0.09$ & $>0.06$ \\
\hline Liu et al. $[19]$ & 20 & $<28$ & 0.04 & $0.04($ approx. $)$ & $>0.06$ \\
\hline Luitel et al. $[14]$ & 20 & $<27$ & 0.291 & 0.270 & $>0.13$ \\
\hline Mandal et al. $[20]$ & 20 & 31.09 & 0.118 & 0.0279 & 0.0904 \\
\hline Najjarzadeh et al. $[17]$ & 33 & $<29$ & $\mathrm{NG}^{*}$ & $\mathrm{NG}^{*}$ & $\mathrm{NG}^{*}$ \\
\hline Present work & 20 & 31.6 & 0.0390 & 0.0260 & 0.0632 \\
\hline
\end{tabular}

* $\quad$ Not Given extensively in the referred literature

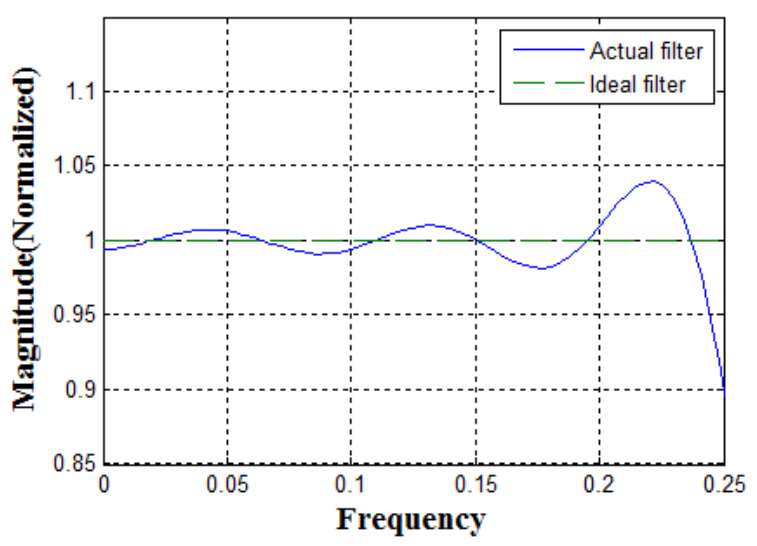

Fig. 2: Passband portion of the magnitude plot

The results of Liu et al. [19], show the maximum stopband attenuation is less than $28 \mathrm{~dB}$, maximum passband ripple is 0.04 , maximum stopband ripple is approximately 0.04 and transition width is much higher than 0.06. However the proposed filter shows performance improvement in every parameter with the maximum stopband attenuation of $31.6 \mathrm{~dB}$, maximum passband ripple of 0.0390 , maximum stopband ripple of 0.0260 and transition width of 0.0632 . Also it can be observed from Table 4 that the simulation results of proposed algorithm are better than the other presented results.

The normalized magnitude response drawn from the optimized filter coefficients from Table 1 is shown in Fig. 1. The plots of both ideal and approximate filter are shown in Fig. 1 for comparative evaluation. The passband ripple of the figure can be studied by looking into the passband region of the designed filter as shown in Fig. 2.

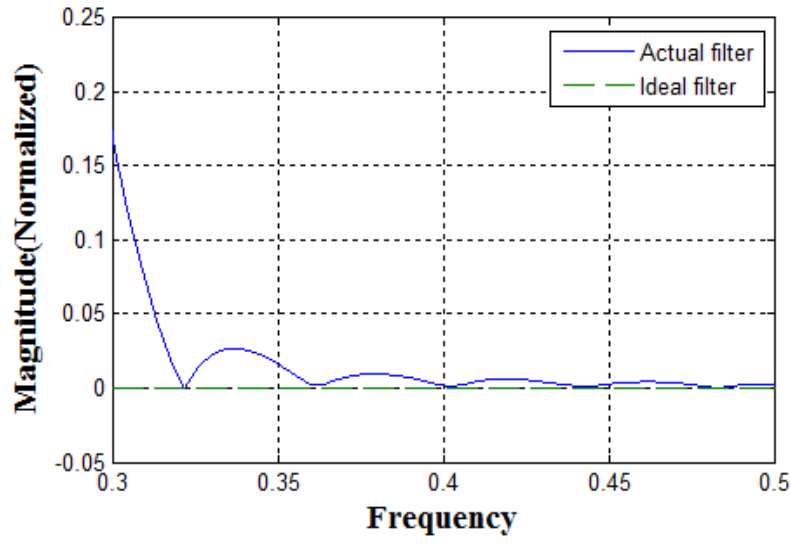

Fig. 3: Stopband portion of the magnitude plot

dB plot of actual filter

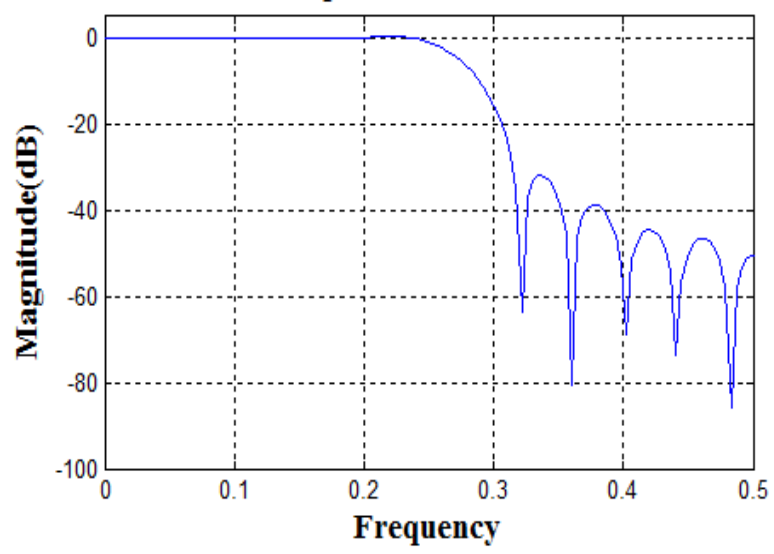

Fig. 4: dB plot for LP FIR filter of order 20 
As can be observed from Fig. 2, the ripples are very small and have the maximum deviation of 0.0390 in terms of magnitude from ideal filter response. Further stopband ripple in Fig. 3 shows maximum deviation of 0.0260 in terms of magnitude from the ideal zero response. The magnitude response in $\mathrm{dB}$ is plotted in Fig. 4 from where the maximum stopband attenuation in terms of $\mathrm{dB}$ is observed to be $31.6 \mathrm{~dB}$. All of the results are summarized in Table 3.

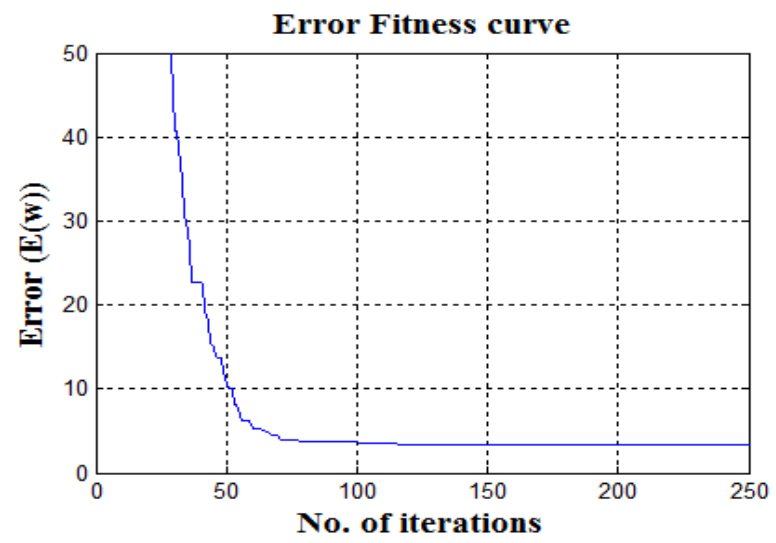

Fig. 5: Convergence curve for LP FIR filter of order 20

The convergence rate of the proposed algorithm is inferred from Fig. 5 showing how efficiently the algorithm converges to global optimal result of minimum error fitness value. The algorithm start converging smoothly after about 100 iteration cycles for all 100 runs case, as observed by the author.

\section{CONCLUSION}

This paper presented the use of Particle Swarm Optimization using constriction factor approach for non-linear, nondifferential, multi-modal LP FIR filter design problem. The proposed method has best convergence characteristics. Comparison of the resulting performance parameters such as maximum passband ripple, maximum stopband ripple and maximum stopband attenuation(in $\mathrm{dB}$ ) with the results reported in past literatures shows significant improvement inferring that the proposed algorithm is best of them all. It is revealed that the proposed algorithm converges to the specified optimum result within very small number of iterations and much less execution time. Thus it has good application prospect in practical filter design problems used in various signal processing systems.

\section{REFERENCES}

[1] J.G. Proakis and D.G. Manolakis, "Digital Signal Processing-Principles, Algorithms and Applications", New Delhi: Prentice-Hall, 2000.

[2] T.W. Parks and C.S. Burrus, "Digital Filter Design". New York:Wiley, 1987.

[3] O. Herrmann and W. Schussler, "Design of non recursive digital filters with linear phase", Electronics Letter, vol. 6, pp.329-330, 1970.

[4] T.W. Parks and J.H. McClellan, "Chebyshev approximation for non recursive digital filters with linear phase”, IEEE Transactions on Circuit Theory, vol. 19, pp. 189-194, 1972.

[5] L.R. Rabiner, "Approximate design relationships for High-pass FIR digital filters", IEEE Transactions on Audio Electroacoustics, vol. 21, pp. 456-460, 1973.
[6] M. B. Joaquim and A.S. Lucietto, "A nearly optimum linear-phase digital FIR filters design”, Digital Signal Processing, vol. 21, pp. 690-693, 2011.

[7] S. Mandal, S.P. Ghoshal, R. Kar and D. Mandal, "Design of optimal linear phase FIR high pass filter using craziness based particle swarm optimization technique", Journal of King Saud University, vol. 24, pp. 83-92, 2012.

[8] H.C. Lu and S.T. Tzeng, "Design of arbitrary FIR log filters by genetic algorithm approach", Signal Processing, vol. 80, pp. 497-505, 2000.

[9] D. Karaboga, D.H. Horrocks, N. Karaboga and A. Kalinli, "Designing digital FIR filters using Tabu search algorithm", IEEE International Symposium on Circuits and Systems, vol.4, pp.2236-2239, 1997.

[10] N. Karaboga, "A new design method based on artificial bee colony algorithm for digital IIR filters", Journal of the Franklin Institute, vol. 4, pp.328-348, 2009.

[11] N. Karaboga and B. Cetinkaya, "Design of Digital FIR Filters Using Differential Evolution Algorithm", Circuits System Signal Processing, vol. 25, pp. 649-660D, 2006.

[12] J.I. Ababneh and M.H. Bataineh, "Linear phase FIR filter design using particle swarm optimization and genetic algorithms", Digital Signal Processing, vol. 18, pp. 657-668, 2007.

[13] J. Kennedy and R. Eberhart, "Particle Swarm Optimization", In Proceeding of IEEE International Conference On Neural Network, vol. 4, pp. 1942-1948, Perth, 1995.

[14] B. Luitel and G.K. Venayagamoorthy, "Differential Evolution Particle Swarm Optimization for Digital Filter Design”, IEEE Congress on Evolutionary Computation, Hong Kong, pp. 3954-3961,2008.

[15] J. Sun, B. Feng and W.B. Xu, "Particle Swarm Optimization with Particles Having Quantum Behaviour", In Proceedings of Congress on Evolutionary Computation, pp. 325-331, 2004.

[16] W. Fang, J. Sun, W. Xu, and Jing Liu, "FIR Digital Filters Design Based on Quantum-behaved Particle Swarm Optimization", First International Conference on Innovative Computing, Information and Control, vol. 1, pp. 615-619, Beijing,2006.

[17] M. Najjarzadeh and A.Ayatollahi, FIR Digital Filters Design: "Particle Swarm Optimization Utilizing LMS and Minimax Strategies", International symposium on Signal Processing and Information Technology, pp. 129132, Sarajevo, 2008.

[18] M. Clerc and J. Kennedy, "The particle swarmexplosion, stability, and convergence in a multidimensional complex space", IEEE Transaction on Evolutionary Computation, vol. 6, pp. 58-73, 2002.

[19] G. Liu, Y.X. Li, and G.He, "Design of Digital FIR Filters Using Differential Evolution Algorithm Based on Reserved Gene", IEEE Congress on Evolutionary Computation, pp. 1-7, Barcelona, 2010.

[20] S. Mondal, D. Chakraborty, R. Kar, D. Mandal and S.P. Ghoshal, "Novel Particle Swarm Optimization for Low Pass FIR Filter Design", WSEAS Transactions on Signal Processing, vol. 8, pp. 111-120, 2012. 